\title{
Green and Orange CdTe Quantum Dots as Effective pH-sensitive Fluorescent Probes for Dual Simultaneous and Independent Detection of Viruses
}

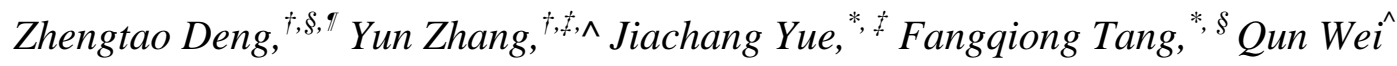

$\uparrow$ These authors contributed equally to this work.

†nstitute of Biophysics, Chinese Academy of Sciences.

$\S$ Technical Institute of Physics and Chemistry, Chinese Academy of Sciences.

TGraduate School of Chinese Academy of Sciences.

${ }^{\wedge}$ Beijing Normal University. 
(1) Quantify the CdTe quantum dots labeled on the outside surface of one chromatophore.

About $12.5 \mu \mathrm{l} \mathrm{BChl}$ was added in $250 \mu \mathrm{l}$ buffer, the density of $\mathrm{BChl}$ was $0.3 \mu \mathrm{g} / \mu \mathrm{l}$; therefore, the total about $37.5 \mu \mathrm{g}$ BChl of chromatophore was used, so that we get about $4 \times 10^{-8} \mathrm{~mol}$. (the BChl molecule weight of BChl was about $890 \mathrm{~mol} / \mathrm{g})$.

It was reported that $6 \mathrm{LH} 1$ contain $600 \mathrm{BChl}$ and $10 \mathrm{LH} 2$ contain $400 \mathrm{BCh},{ }^{1,2}$ therefore the total BChl molecule number was get:

$$
4 \times 10^{-8} \mathrm{~mol} \times 6.02 \times 1023=2.4 \times 10^{16}
$$

Furthermore, one chromatophore was consisted with 16 LH1 and 50 LH2, 29,30 so that the one chromatophore was total about BChl molecular number was bout $3800 \mathrm{BChl}$. From above we get the total chromatophores numbers as follow:

$$
2.4 \times 10^{16} / 3800=6.3 \times 10^{12} \text {. }
$$

Then the concentration was about CdTe quantum dots is $1 \times 10^{16}$ particles $/ \mathrm{ml}$, and used $100 \mu \mathrm{l}$ solution of CdTe quantum dots, so that the total CdTe quantum dots number was $1 \times 10^{15}$. Due to the fraction of attached to chromatophores was $60 \%$, so we get attached on chromatophores-CdTe quantum dots number was followed: 
$1 \times 10^{15} \times 60 \%=6 \times 10^{14}$

Furthermore calculating how much CdTe quantum dots was attached on one chromatophore was followed:

$6 \times 10^{14} / 6.3 \times 10^{12}=100$

(2) The fluorescence intensity of $535 \mathrm{~nm}$ CdTe quantum dots-chromatophores changes during ATP synthesis/hydrolysis.

The relationship between the Fluorescence intensity and synthesis/hydrolysis of CdTe quantum dots-chromatophores was showed in the Fig.S2. Fig.S2 showed that the fluorescence intensity was increased when the ATP synthesis, and the fluorescence intensity was decreased when the ATP the hydrolysis.

\section{(3) Effect of inhibitor on fluorescence intensity changes of the QDs-labeled chromatophores.}

DCCD is $\mathrm{F}_{0}$ channel inhibitor, which can inhibit the ATPase activity of chromatophores and block the proton pump; therefore, it can be used to confirm the fluorescence intensity change of the QDs was really caused by the proton flux out of the chromatophores. As shown in Fig.S3, When $4 \mathrm{mM}$ ADP was added to initialize reaction at $37^{\circ} \mathrm{C}$, the fluorescence intensity increased, which indicated an increase of $\mathrm{pH}$ value nearby chromatophores (curve a); whereas, QDs-labeled chromatophores incubated with $2 \mu \mathrm{M}$ DCCD prior showed no obvious change of fluorescence 
intensity(curve b). The fluorescence intensity of QDs-labeled chromatophores changes without adding ADP as the control. Because of the lack of ADP, ATP can't be synthesized and few protons were pumped out of chromatophores, consequently, the fluorescence of QDs had no obvious changes (curve c). The results showed that the increasing of fluorescence intensity was coupled with ATP synthesis activity and proton flux.

\section{(4) The ATP synthesis activity was measured with different system.}

The ATP synthesis activity was measured by the luciferin-luciferase method. Fig.S4 group (A) showed that the $\mathrm{F}_{0} \mathrm{~F}_{1}$-ATPase (column a) and $\mathrm{F}_{0} \mathrm{~F}_{1}$-ATPase with $\mathrm{H} 9$ virus (column $\mathrm{b}$ ) was not different. The Fig.S4 group (B) showed that the ATP synthesis activity was not different whether labeled with CdTe quantum dots (column c) or without labeled (column d).

\section{References}

(1) Tihamér, G.; Volkhard, H. Biophys. J. 2006, 91, 921-926.

(2) Svetlana, B.; Raoul, N. F.; Alistair, C. S.; John, D. O.; Kees, O. W.; Rienk, G.; Robert, A. N;

Bullough, P. A.; Otto1, C.; Neil, H. C. Nature 2004, 403, 1058-1062. 


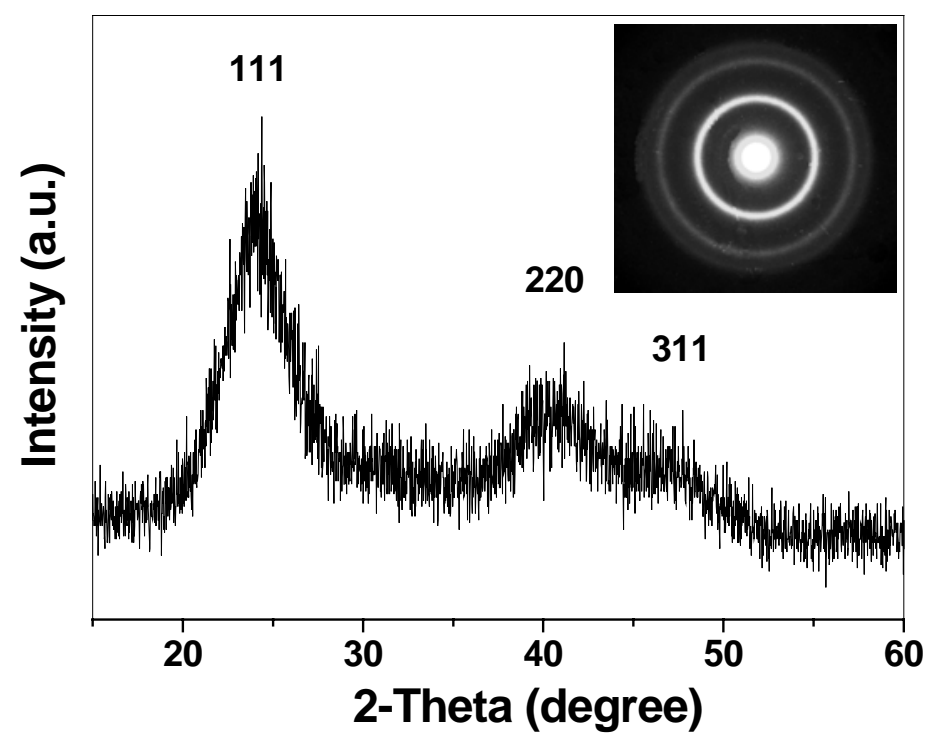

Figure S1. Typical XRD pattern of the CdTe QDs show in Figure 1; Inset: the corresponding SAED pattern of the CdTe QDs show in Figure 1. 


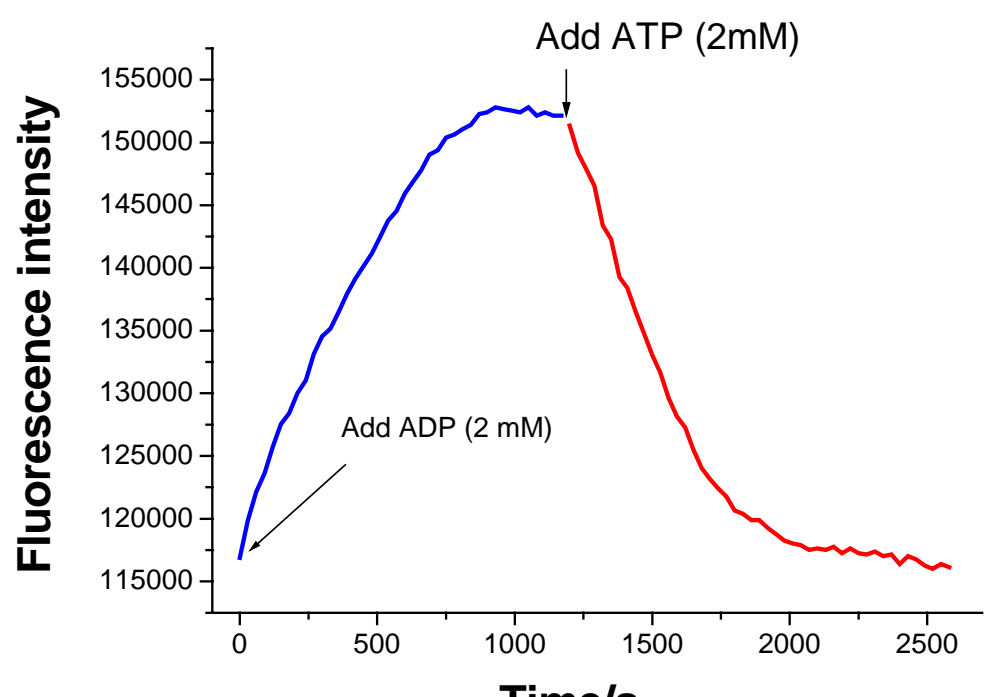

Figure S2. The fluorescence intensity of CdTe QDs on chromatophores.

When adding ADP synthesis buffer, the fluorescence intensity was increase during time course 1200s, then in replace the buffer, adding ATP hydrolysis buffer, the fluorescence intensity was decreased, during time course 1200s. The sample was fixed on the surface of the glass with BPCL-III (LED). 


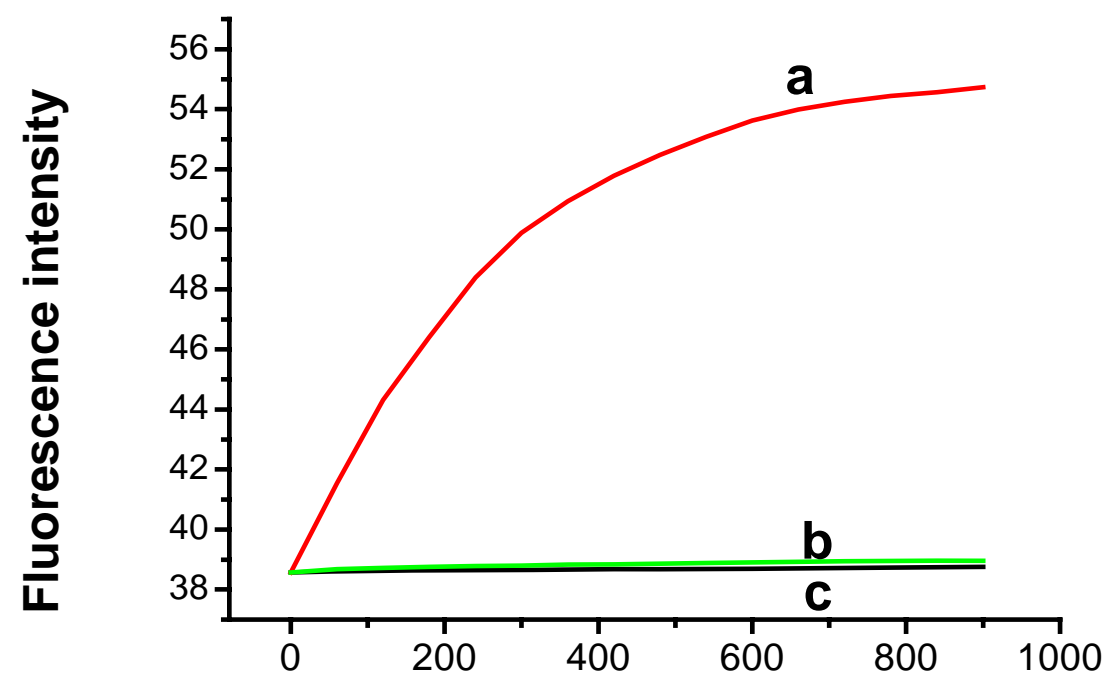

Time/s

Figure S3. Curve (a), fluorescence intensity changes of QDs-labeled chromatophores when 4 mM ADP was added to initialize reaction; Curve (b), QDs-labeled chromatophores incubated with $2 \mu \mathrm{M}$ DCCD for $1 \mathrm{~h}$ at room temperature prior when $4 \mathrm{mM}$ ADP was added to initialize reaction; Curve (c), fluorescence intensity changes of QDs-labeled chromatophores without adding ADP as control. 


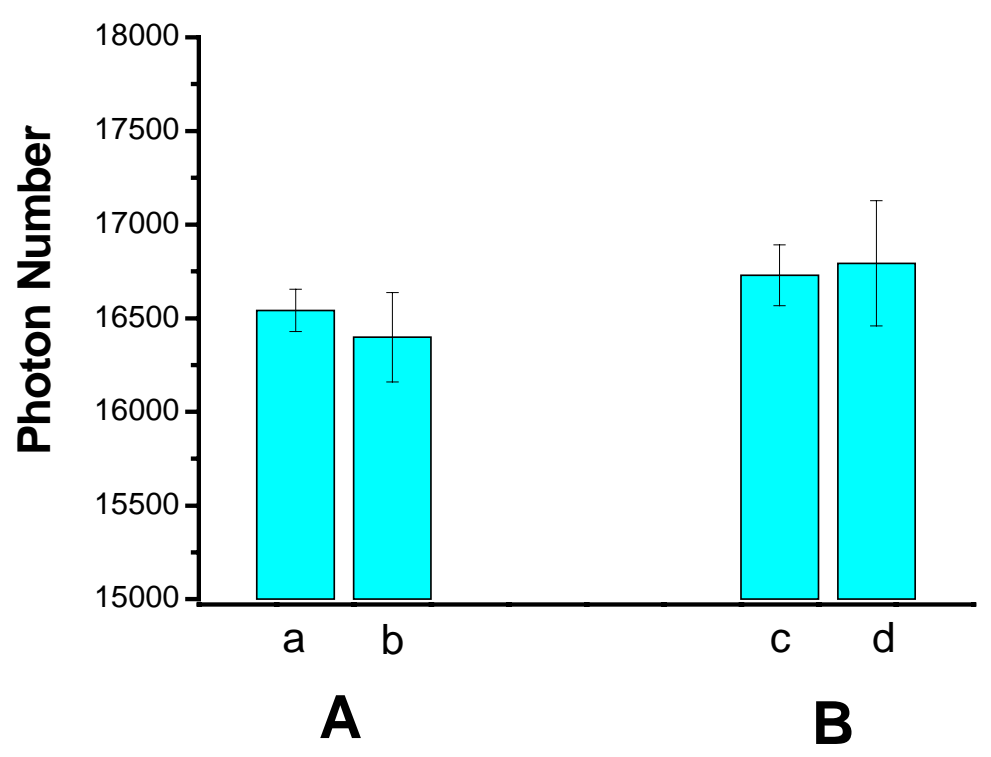

Figure S4. The ATP synthesis activity of $\mathrm{F}_{0} \mathrm{~F}_{1}$-ATPase was measured. Group A: column (a) $\mathrm{F}_{0} \mathrm{~F}_{1}$-ATPase within chromatophores as a control; column (b) $\mathrm{F}_{0} \mathrm{~F}_{1}$-ATPase within chromatophores with $\mathrm{H} 9$ virus. Group B: column(c) $\mathrm{F}_{0} \mathrm{~F}_{1}$-ATPase within chromatophores as a control; column (d) $\mathrm{F}_{0} \mathrm{~F}_{1}$-ATPase within chromatophores labeled with CdTe quantum dots. 


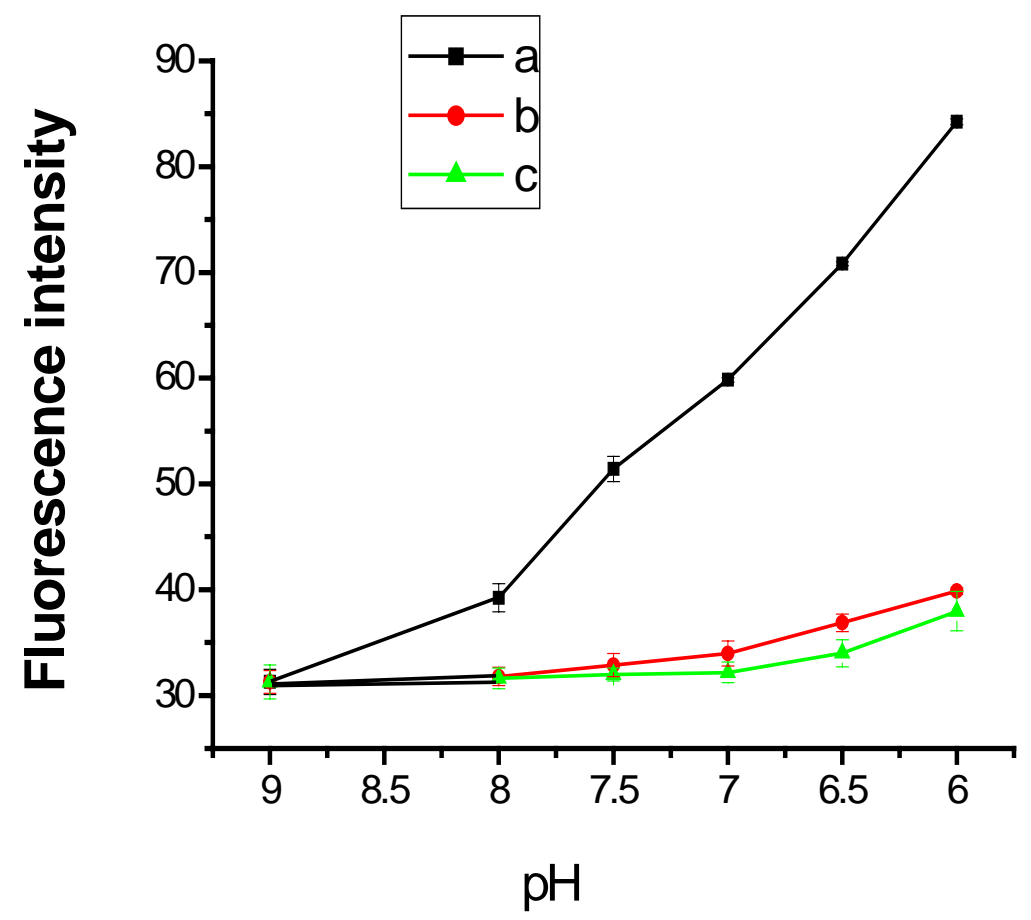

Figure S5. Photoluminescence intensity data of the green CdTe QDs under various $\mathrm{pH}$ values. Curve (a): The free green CdTe QDs in buffer; Curve (b) The green CdTe QDs on-chromatophores in buffer; Curve (c) The green-QDs-biosensors in buffer under LED excitation. 